\title{
A new piezoelectric microphone with divided electrodes and its applications
}

\author{
Nobuomi Imai \\ Primo Co., Ltd., \\ 6-25-1, Mure, Mitaka, 181 Japan
}

(Received 12 December 1989)

\begin{abstract}
The improvement in performance of the microphone using piezoelectric creamic which includes the FET circuit, is described in this research report. The most important point in the design of the microphone is how to improve the transduction efficiency. This paper analyzes a microphone as a system consisted of a number of subsystems. Suppression of the energy transmission loss between these subsystems is an important requirement in designing the microphone. This paper also solves this problem by dividing the electrode of the piezoelectric ceramic and connecting the divided electrodes in series. A large output is obtained by input of high voltage into the FET which has voltage drive characteristics. High performance and stability were obtained by this microphone together with the use of ceramic for the entire diaphragm system in the prototype microphone. The performance characteristics of this prototype microphone are Sensitivity: $-35.8 \mathrm{~dB} / \mathrm{Pa} 1 \mathrm{kHz}$, Electrostatic capacitance: $275 \mathrm{pF}$, Frequency response: $\pm 1.5 \mathrm{~dB}$ at $20 \mathrm{~Hz}$ to $12.5 \mathrm{kHz}$.
\end{abstract}

Keywords: Piezoelectric microphones, Divided electrodes, Dome type diaphragm, Piezoelectric sensing devices

PACS number: 43. 88. Fx

\section{INTRODUCTION}

Since piezoelectric ceramic is capacitive and generally has high impedance, considerable missmatching occurs when it is connected directly to conventional integrated circuits or transistor circuits. It is clear that impedance transduction is essential for practical applications.

For this reason, positive use of FET circuits is necessary. However, a simple connection of the piezoelectric ceramic and the FET circuit only results in miss-matching so that only a part of the power can be output.

Therefore, the author devised the most suitable conditions for proper matching with the FET circuits by division of the electrode of piezoelectric ceramic and series connection of the divided electrodes to obtain high voltage and high impedance. Owing to this design, the optimum matching condi- tions can be set by division of the electrode into the appropriate number of segments although the piezoelectric ceramic may vary in shape. As the result, the output level was increased greatly (increase of about $20 \mathrm{~dB}$ in practical applications) and the transduction efficiency was also improved.

In case of the application example of the microphone, a thin film of $\mathrm{Y}_{2} \mathrm{O}_{3} \mathrm{PSZ}$ (Partially Stabilized Zirconia, $\mathrm{Y}_{2} \mathrm{O}_{3} 3 \mathrm{~mol} \%$ doped) was formed into a dome shape for the base material of the diaphragm. A ring shaped piezoelectric ceramic was bounded on this base to secure a flat frequency response, expansion of the high end and stability.

\section{DESIGN PRINCIPLE OF DIVIDED ELECTRODES SYSTEM}

The following four characteristics were considered to obtain the optimum matching condition with FET circuits. 
1) Boosting characteristics with use of divided electrodes of piezoelectric diaphragm.

2) Insertion loss characteristics of FET circuit.

3) Temperature characteristics of piezoelectric ceramic.

4) Noise level of FET (ircuit. ${ }^{1)}$

\subsection{Principle of Divided Electrodes ${ }^{3,6}$ )}

The most simple form is shown in Fig. 1. When the vibration of the piezoelectric ceramic is in the diametrical direction, $e$ is generated at points $\mathrm{a}$ and $\mathrm{b}$ in case of the former type (a). In case of type (b), however, $2 e$ is generated at points a and $\mathrm{b}$, because the electrode is divided into 2 parts and they are connected in series. When the electrode is divided into $n$ segments, we can obtain an equivalent circuit having an internal impedance of $n^{2} z_{1}$ and source voltage of $n e$.

If the thickness of each element is the same in case of the poling method, $2 q \mathrm{~V}$ should be applied between $\mathrm{a}$ and $\mathrm{b}$ for type (b) when voltage $q V$ is required between $\mathrm{a}$ and $\mathrm{b}$ for type (a). Therefore, $n q V$ is required in case the electrode is divided into $n$ number of segments.

\subsection{Calculation of Dividing Number}

The typical FET circuit is shown in Fig. 2(a). This can be analyzed as Fig. 2(b). Since the input impedance of the FET circuit is determined by only the electrostatic capacitance $C_{\mathrm{g}}$ between the gate and source, the equivalent circuit of only the input side which includes this $C_{\mathrm{g}}$ is shown in Fig. 2(c). The following equation is established.

$$
\left[\begin{array}{c}
v_{1} \\
i_{1}
\end{array}\right]=\left[\begin{array}{cc}
1+n^{2} z_{1} / z_{2} & n^{2} z_{1} \\
1 / z_{1} & 1
\end{array}\right]\left[\begin{array}{c}
v_{2} \\
i_{2}
\end{array}\right]
$$

Where $z_{1}=-j / \omega C_{0}$ and $z_{2}=-j / \omega C_{\mathrm{g}}$. Therefore,

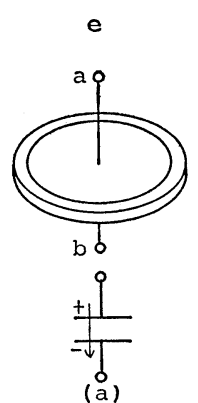

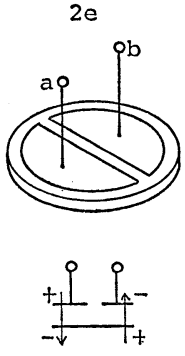

(b)

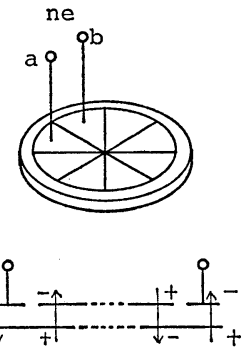

(c)
Fig. 1 Principle of divided electrodes.

$$
v_{2}=n e /\left(1+n^{2} z_{1} / z_{2}\right)
$$

Here, the condition for the maximum value of $v_{2}$ can be obtained from $d v_{2} / d n=0$. As the solution, we obtain,

$$
n=\sqrt{\frac{C_{0}}{C_{\mathrm{g}}}}
$$
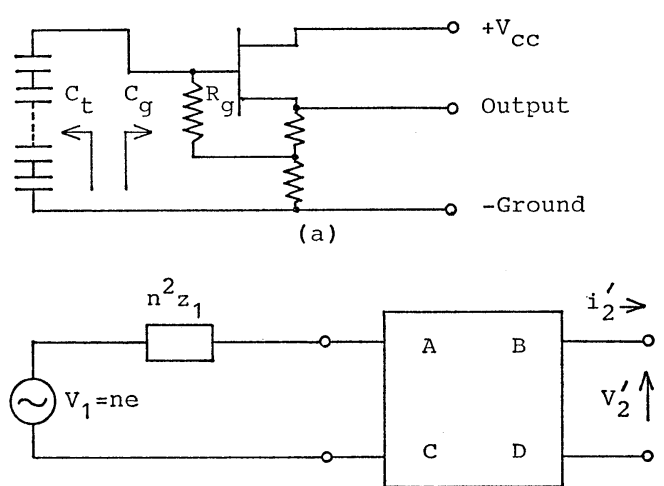

(b)

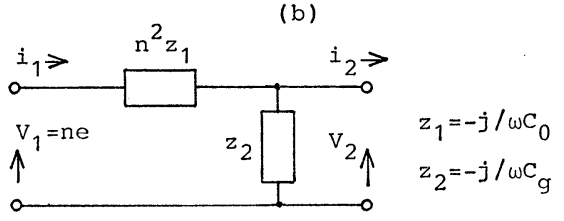

(c)

Fig. 2 Typical FET circuit and equivalent circuits.

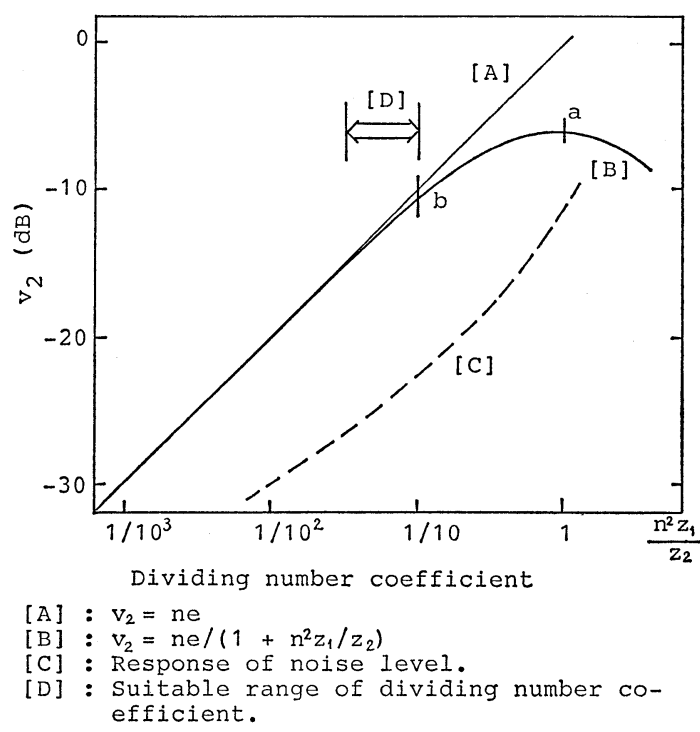

Fig. 3 Relation of dividing number coefficient to $v_{2}$ and noise level of FET circuit. 


\section{N. IMAI: PIEZOELECTRIC MICROPHONES}

Provided that, $C_{0}$ is normally an order of $1 \times$ $10^{4} \mathrm{pF}, C_{\mathrm{g}}$ is around $10 \mathrm{pF}$ for the $N$-channel junction type. In conclusion, $v_{2}$ becomes maximum at the condition of Eq. (3). At the same time, the output power of the FET circuit also becomes maximum. These are expressed in graph form in Fig. 3.

\subsection{Examination of Temperature Characteristics of Capacitance in Piezoelectric Ceramic}

The electrostatic capacitance varies according to the temperature because the relative dielectric constant of the piezoelectric ceramic has temperature characteristics. For this reason, $v_{2}$ in Fig. 4(a) must be considered. The relation between the temperature and the electrostatic capacitance is expressed by the following equation.

$$
C=C_{\mathrm{a}}(1+0.0063 t)
$$

Here, $C_{\mathrm{a}}$ is the $C$ at $0^{\circ} \mathrm{C}$ and $t$ is temperature. Figure 4(a) becomes, $v_{2}=v_{1} C_{\mathrm{t}} /\left(C_{\mathrm{g}}+C_{\mathrm{t}}\right)$. The derived function is expressed as $d v_{2} / d C_{\mathrm{t}}=-v_{1} C_{\mathrm{t}} /\left(C_{\mathrm{g}}+C_{\mathrm{t}}\right)^{2}$. The derivative at point a in Fig. 4 is $1 / 4$. On the other hand, the derivative at point $b$ is $1 / 12.1$. This point $b$ corresponds to point $b$ in Fig. 3. This means that the effect of the variation in the electrostatic capacitance due to temperature is far smaller at point $b$.

\subsection{Examination of Noise Level Characteristics}

For designing the microphone, we must, of course, select FET of low noise characteristics. However, the gate resistor $r_{\mathrm{g}}$ and the input capacitance $C_{\mathrm{t}}$ (see Fig. 2(a)) are also factors which must be considered in respect to the noise level. The noise level is lower when they are larger. Since the time constant is also determined by these two, they cannot be made too large. Particularly, the noise level rises rapidly when $C_{\mathrm{t}}$ becomes comparable to $C_{\mathrm{g}}$. For this reason, point a is unsuitable in respect to

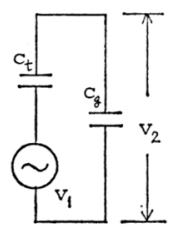

(a)

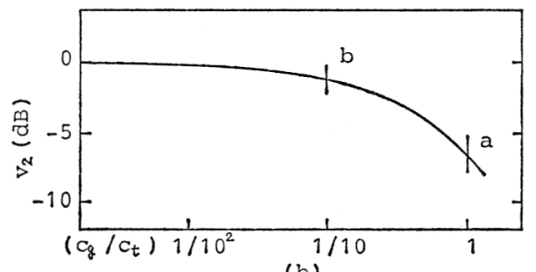

(b)
Fig. 4 Insertion loss characteristics of FET circuit. the signal to noise ratio. The dotted line $[\mathrm{C}]$ in Fig. 3 indicates the typical response of the noise level. The vicinity of point $b$ is better in respect to the signal to noise ratio also.

\section{APPLICATION EXAMPLE}

\subsection{Designing of Diaphragm}

The dome type diaphragm was used for the trial manufacture of the microphone. This microphone was designed for application as a sensor for instrumentation and for monitoring. Priority was placed on high performance, high stability and high durability. Particularly, the dome type diaphragms are generally considered to have the following features.

1) Since it is a single resonance system, smooth reproduction of the high frequencies is possible.

2) It has structural stability.

3) Since the mechanical impedance is small, the transduction efficiency is high and damping of the primary resonance is easy.

In order to make the best use of these features of the dome type, $\mathrm{Y}_{2} \mathrm{O}_{3} \mathrm{PSZ}$ ceramic was used for the

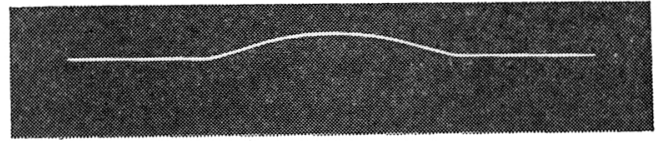

(a) Photograph of cross section of formed material.

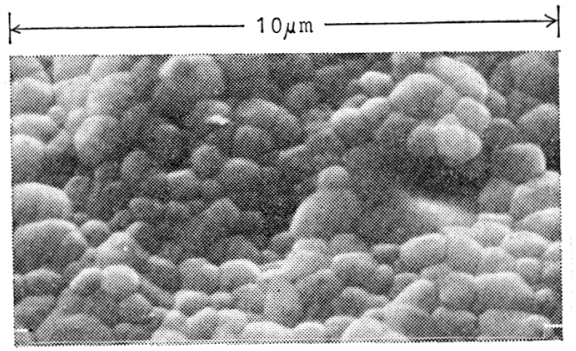

(b) SEM photograph of surface.

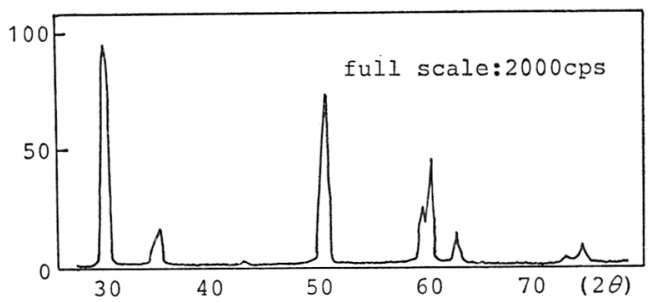

(c) X-ray diffraction chart of $\mathrm{Y}_{2} \mathrm{O}_{3} \cdot \mathrm{PSZ}$ ceramic. Fig. 5 Evaluation of $\mathrm{Y}_{2} \mathrm{O}_{3} \mathrm{PSZ}$ ceramic. 
base meterial of the diaphragm.

The evaluation of this material is shown in Fig. 5. Figure 5(a) is a photograph of the cross section of the formed material. Figure 5(b) is an SEM photograph of the surface. It can be clearly seen that the grains are uniform in size of less than $1 \mu \mathrm{m}$. Figure 5 (c) is the X-ray diffraction chart. It was confirmed from these examination result that it was good sintered material of $\mathrm{Y}_{2} \mathrm{O}_{3}$ PSZ.

On the other hand, the piezoelectric ceramic was processed into a ring shape and bonded onto the base material. The outer diameter of the dome part and inner diameter of the ring were made the same. The electrode was divided into ten parts. Silver was used as the electrode material and it was baked at $800^{\circ} \mathrm{C}$.

The structure and characteristics of this diaphragm $^{2,4,5)}$ are given in Fig. 6. In Fig. 6(b), an $f_{02}$ value which is approximately 5 times that of $f_{01}$ was obtained in case of the free edge. This is considered to be the normal value. It can be deduced that no resonance of a higher order exists between $f_{01}$ and $f_{02}$.

In Fig. 7, the diaphragm which was assembled as a microphone capsule, was driven in a free field to measure the voltage generating condition in each part of the piezoelectric diaphragm. Four electrodes are provided on a concentric circle for the piezoelectric ceramic. It can be seen from the result, that the whole diaphragm is activated by the low frequency $(1 \mathrm{kHz})$ and that only the dome and its rising part are mainly activated by the high frequency $(20 \mathrm{kHz})$.

Figure 8 shows the temperature characteristics of the resonance frequency of the diaphragm unit in comparison with the round flat type diaphragm. Since the round flat type is weak against bending, it can be considered that a strain is applied due to the difference in the coefficient of the thermal expansion between the base material and the piezoelectric ceramic. On the other hand, it shows that the dome type has rigidity.

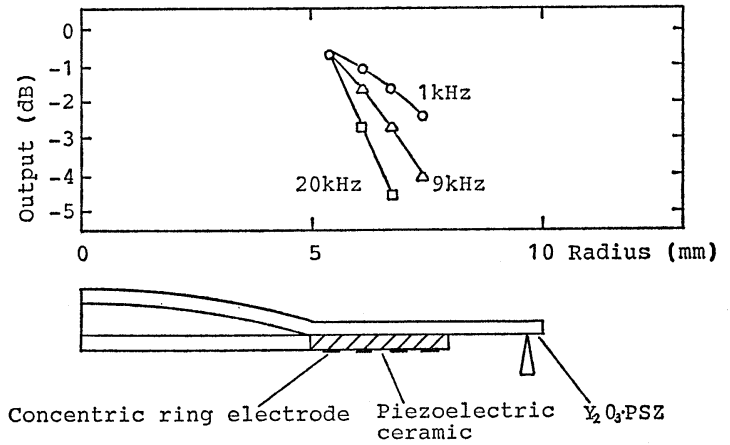

Fig. 7 Voltage distribution of diaphragm.

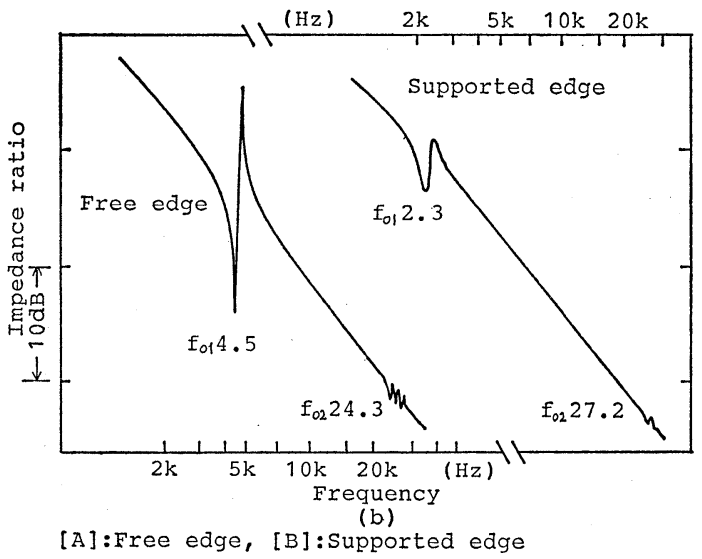

Fig. 6 Physical dimensions and impedance characteristics of diaphragm.

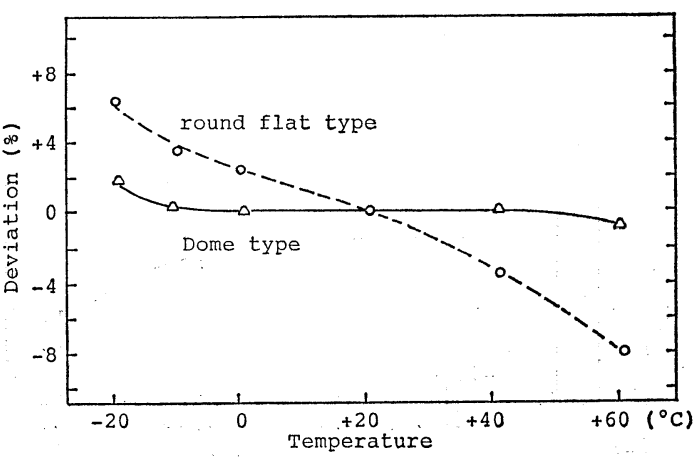

Fig. 8 Temperature characteristics of resonance frequency of diaphragm. 


\section{N. IMAI: PIEZOELECTRIC MICROPHONES}

\subsection{Structure of Microphone Capsule}

The acoustic structure, equivalent circuits and sectional view of the prototype microphone capsule are shown in Fig. 9. The basic configuration of the acoustic circuits is the same as the conventional types. The first front chamber is provided in front of the diaphragm. The first back chamber, acoustic resistance and second back chamber are arranged in order behind the diaphragm.

The supporting structure of the diaphragm is in Fig. 10. A knife edge type support is used to optimize the peripheral supporting structure. An RTV (Room Temperature Vulcanizing) type silicon rubber is used to fix the periphery of the diaphragm.

Figure 11 shows how the electrical impedance of

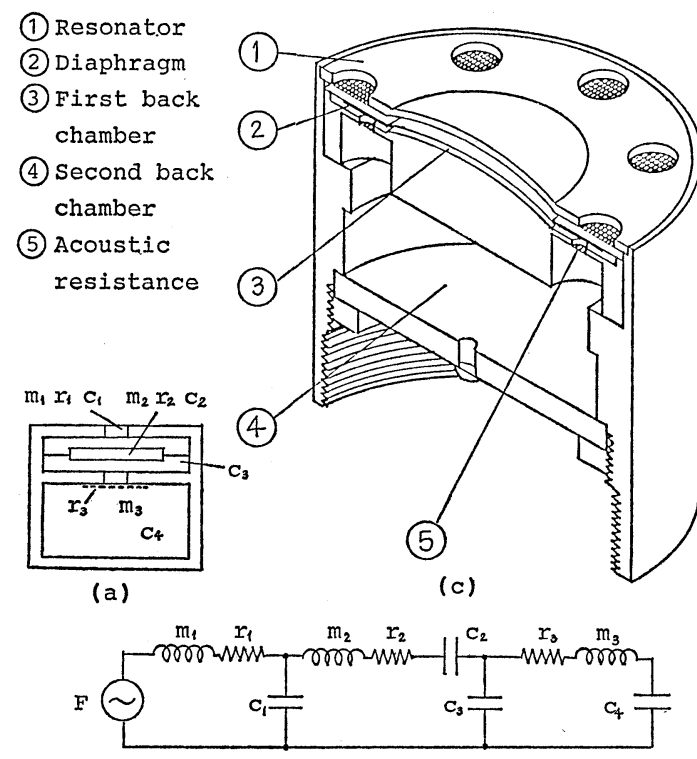

(b)

Fig. 9 Acoustic structure, equivalent circuit and sectional view of prototype microphone capsule. the capsule varies when the constants of the acoustic circuits are varied. The symbols in Fig. 11 correspond to those used in Fig. 9(a). In case of the open characteristics, no acoustic circuits are provided in the front or back of the diaphragm. In case of $c_{3}+c_{4}$ characteristics, the first and second back chambers are combined. In case of the $c_{3}$ characteristics, the $r_{3}$ hole is blocked. When the effective mass of the diaphragm is $m_{1}$ and its compliance is $c_{\mathrm{x}}$, the resonance frequency can be expressed as follows.

$$
f_{\mathrm{x}}=1 /\left(2 \pi \sqrt{m_{1} c_{\mathrm{x}}}\right)
$$

When the corresponding $c_{\mathrm{x}}$ of $f_{\mathrm{x}}$ in Fig. 11 is substituted in Eq. (5) with $c_{2}: 1.00$ as the base for calculation, we obtain $c_{3}: 0.43$ and $c_{4}: 7.57$.

\subsection{Performance of Prototype Microphone Capsule} The technical data of the microphone capsule are

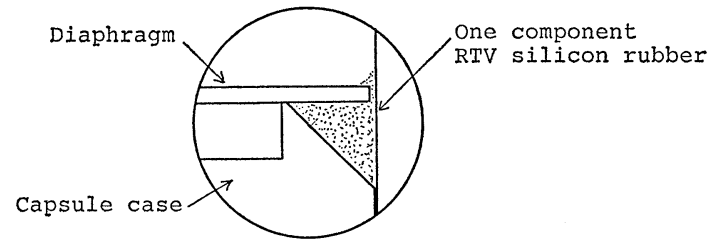

Fig. 10 Supporting structure of diaphragm.

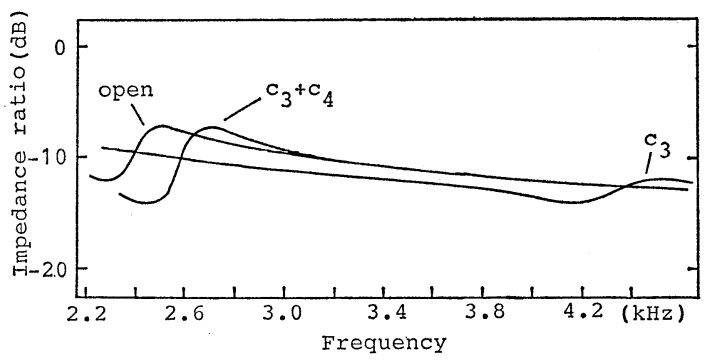

Fig. 11 Changing of resonance frequency by changing of acoustic chambers.

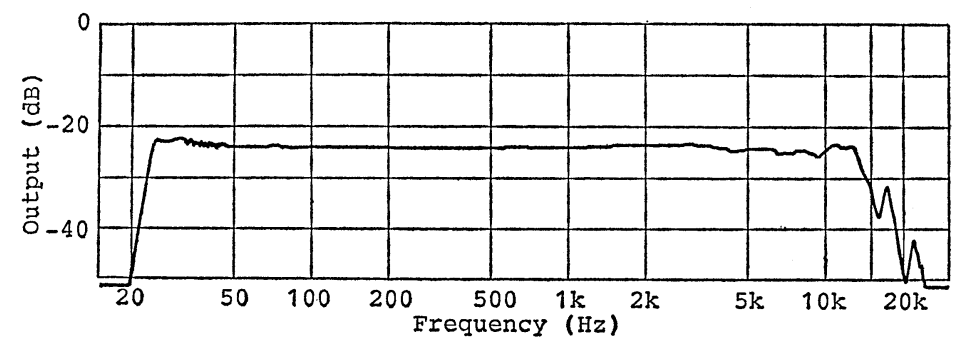

Fig. 12 Frequency response of prototype microphone capsule (Free field $0^{\circ}$ ). 


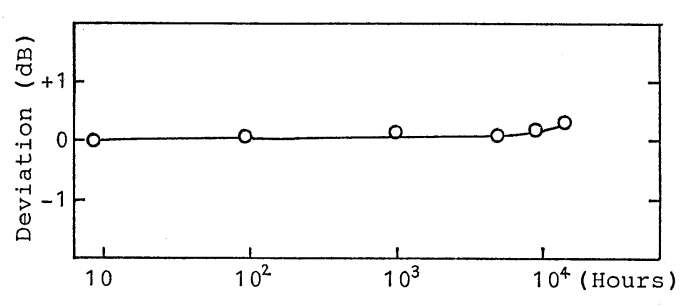

Fig. 13 Aging characteristics of prototype microphone capsule $(1 \mathrm{kHz})$.

shown in below.

$\begin{array}{ll}\text { Outer diameter } & 23.77 \mathrm{~mm} \\ \text { Directional pattern } & \text { Omni-directional } \\ \text { Sensitivity } & -35.8 \mathrm{~dB} / \mathrm{Pa} 1 \mathrm{kHz} \\ \text { Electrostatic capacitance } & 275 \mathrm{pF}\left(20^{\circ} \mathrm{C}\right) \\ \text { Noise level } & 15 \mathrm{~dB} \text { Equivalent } \\ & \begin{array}{l}\text { input conversion } \\ \text { value (A-weighted). }\end{array} \\ \text { Frequency response } & \text { Given in Fig. 12. } \\ \text { Aging characteristics } & \text { Given in Fig. 13. }\end{array}$

\section{DISCUSSION}

Regarding the determination of the divided electrodes number, the range indicated by the arrow mark [D] $\left(n^{2} z_{1} / z_{2}\right.$ is the range of $3.1 / 10^{2}$ to $\left.1 / 10\right)$ and its vicinity in Fig. 3 will be the most suitable range of the dividing number coefficient. It should be set on the right side of this range when a large output voltage is desired. On the other hand, it should be set on the left side of this range when the stability and signal to noise ratio are desired. In case of an application for detection of a very small signal with good signal to noise ratio, it should be set near $n^{2} z_{1} / z_{2}$ $=1 / 10$. In case of an application for a large input, clipping of the FET circuit can be prevented by reducing the dividing number $n$. In case of a normal microphone, the appropriate dividing number is about ten.

In the calculation of the dividing number $n$, there is a loss between patterns according to the dividing pattern (see Fig. 6(a)). Therefore, the capacitance which corresponds to this area should be subtracted beforehand from $C_{0}$ for more accurate calculation.

The factors which will influence the suitable dividing number are the electrode capacitance $C_{0}$ of the piezoelectric ceramic before the division and the electrostatic capacitance $C_{\mathrm{g}}$ between the gate and source of the FET. Therefore, in case the output voltage $v_{2}$ is to be made large, the element capacitance $C_{0}$ should be made as large as possible, and $C_{\mathrm{g}}$ of the FET should be made as small as possible.

\section{CONCLUSION}

The improvement in performance of the piezoelectric microphone is described in this research report. This microphone features the following strong points in comparison with the conventional piezoelectric microphones.

1) Higher sensitivity and better signal to noise ratio are realized. The divided electrode system enables setting of the electrical matching with the FET circuit at the optimum conditions.

2) The stability of performance (including longterm stability) is improved by use of ceramic for the entire diaphragm.

3) Flat frequency response is obtained over a wide range by reduction of the mechanical impedance through the use of a dome type design with thin film ceramic.

These new techniques can be applied, not only to microphones, but also to all sensing devices in which piezoelectric ceramic is used. Particularly, the divided electrode system has the following advantages.

By varying the shape of the piezoelectric ceramic, it can be made to match the mechanical impedance of various media. Even when the piezoelectric ceramic may vary in shape in this case, the electrical matching with the FET circuit can be set at the optimum conditions by dividing the electrode into the appropriate number of segments. This is also effective for miniaturization in designing.

In addition to microphones, application of this design can also be considered for AE sensors, ultrasonic sensors, hydrophones and acceleration sensors.

\section{REFERENCES}

1) A. Mizoguchi, "Analysis of the miniaturization of directional condenser microphone," J. Acoust. Soc. Jpn. (J) 31, 310-317 (1975) (in Japanese).

2) Y. Ichinose and M. Tobita, "Design of piezoelectric bimorph diaphragm," J. Acoust. Soc. Jpn. (J) 42, 441-446 (1986) (in Japanese).

3) N. Imai, "A new piezoelectric microphone with divided electrodes," 2nd Jt. Meet. ASA \& ASJ, W4 (1988).

4) N. Imai, "An application of high sensitivity ceramic microphone incorporating multiple poling method," IEICE Tech. Rep. EA88-1 (1988) (in Japanese).

5) N. Imai, "Designing of wide range piezoelectric 


\section{N. IMAI: PIEZOELECTRIC MICROPHONES}

microphones," IEICE Tech. Rep. EA89-101 (1990) (in Japanese).

6) N. Imai, U.S. Patent, No. 4,559,418 (1985.12).

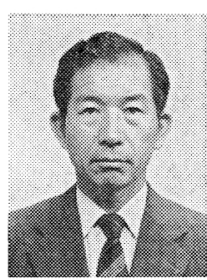

Nobuomi Imai was born in Tokyo, 1935. Graduated from The University of Electro-Communications (1958). He has been working on transducer designs and piezoelectric ceramic materials. R \& D Director of Primo Co., Ltd. A member of the Ceramic Society of Japan and the Acoustical Society

of Japan. 